\title{
Multiquark-Adequate QCD Sum Rules: the Case of Flavour-Exotic Tetraquarks
}

\author{
Wolfgang Lucha ${ }^{1, *}$, Dmitri Melikhov ${ }^{1,2,3, * *}$, and Hagop Sazdjian ${ }^{4, * * *}$ \\ ${ }^{1}$ Institute for High Energy Physics, Austrian Academy of Sciences, Nikolsdorfergasse 18, \\ A-1050 Vienna, Austria \\ ${ }^{2}$ D. V. Skobeltsyn Institute of Nuclear Physics, M. V. Lomonosov Moscow State University, \\ 119991 Moscow, Russia \\ ${ }^{3}$ Faculty of Physics, University of Vienna, Boltzmanngasse 5, A-1090 Vienna, Austria \\ ${ }^{4}$ Institut de Physique Nucléaire, Université Paris-Sud, CNRS-IN2P3, Université Paris-Saclay, \\ 91405 Orsay, France
}

\begin{abstract}
Frequently, theoretical discussions of multiquark hadron states prove to be contaminated or even dominated by contributions of conventional hadrons. For the approach to QCD bound states in terms of QCD sum rules, we show how to get rid of all unwanted ordinary-hadron ballast by boiling down the traditional QCD sum-rule formalism to the nonconventional aspects in the focus of interest.
\end{abstract}

\section{Incentive and Findings: Rigorous Adequacy of Multiquark Studies}

The notion of multiquark hadrons comprises each bound state composed of a larger number of quarks than any ordinary quark-antiquark meson or ordinary three-quark baryon calls its own. In view of the slowly but steadily increasing evidence for the actual experimental observation, and consequently unambiguous confirmation of the existence, of tetraquarks and pentaquarks, we recently embarked on a rigorous theoretical investigation of multiquarks [1-8]. A standard tool for an extraction of properties of hadronic bound states from the underlying quantum field theory, quantum chromodynamics (QCD), is offered by a technique called QCD sum rules [9].

QCD sum rules represent a nonperturbative approach to bound states of quarks and gluons (the degrees of freedom of QCD) in form of analytic relationships between observable hadron properties and the parameters of QCD (strong coupling and quark masses). They can be found upon evaluation of correlation functions of hadron interpolating operators, defined in terms of quark and gluon fields, at both phenomenological, i.e., hadronic, and QCD levels, by insertion of complete sets of hadron states, conversion of nonlocal operator products into series of local operators by exploiting the operator product expansion [10], removal of subtraction terms and suppression of hadron contributions above ground states by applying Borel transformations to new variables, and mutual cancellation of hadronic and perturbative QCD contributions above Borel-variable-dependent effective thresholds [11-13]. The final outcome comprises, at QCD level, both purely perturbative contributions (conveniently represented by dispersion integrals of spectral densities) and nonperturbative contributions, parametrized by vacuum condensates and nicknamed "power corrections" (since getting multiplied by powers of Borel parameters).

\footnotetext{
*e-mail: Wolfgang.Lucha@oeaw.ac.at

**e-mail: dmitri_melikhov@gmx.de

***e-mail: sazdjian@ipno.in2p3.fr
} 
Given the above general recipe, it is, in principle, straightforward to derive QCD sum rules from $n$-point Green functions of (appropriately defined) hadron interpolating operators. In the case of all multiquark hadrons, however, due care has to be paid to the proper disentanglement of those contributions to any such correlation function that may involve the kind of multiquark states of interest and those contributions that are definitely not related to the states under study since, for instance, they affect exclusively ordinary hadrons $[5,6,8]$. More precisely, from our point of view all the latter contributions should be unambiguously identified, stripped off from any QCD sum rule found in the first step by naïve application of the formalism, and discarded.

In the following, for the sake of illustration, let us focus to the particular case of tetraquark mesons contributing in form of intermediate states to some scattering of two ordinary mesons. Quite generally, indices $a, b, c, \ldots$ will label the flavour quantum numbers of the (anti)quarks.

Any contribution of the first kind, i.e., presumably capable of providing information about tetraquarks in the focus of interest, is termed tetraquark-phile [2, 4]: retaining exclusively that sort of contributions entails QCD sum rules enjoying the wanted tetraquark adequacy $[5,6,8]$. Phrased, a little bit more technically, in terms of Feynman diagrams, in order to be regarded as tetraquark-phile a Feynman diagram should depend on the appropriate Mandelstam variable $s$ in a non-polynomial manner and must develop a branch cut starting at a branch point $\hat{s}$ defined by the square of the sum of the masses $m_{a}, m_{b}, m_{c}, m_{d}$ of all involved (anti)quarks $\bar{q}_{a}, q_{b}, \bar{q}_{c}, q_{d}$ acting as constituents of the envisaged tetraquark bound state [1]: $\hat{s}=\left(m_{a}+m_{b}+m_{c}+m_{d}\right)^{2}$. The presence or absence of such cuts is easily verified by application of the Landau equations [14]. Thus established cuts betray the diagram's support of adequate four-quark intermediate states. The latter, in turn, may contribute to the development of a pole related to a physical tetraquark.

Mutatis mutandis, our concepts [5] of sharpening the traditional QCD sum-rule formalism apply, rather generally, to arbitrary kinds of multiquark hadrons. Hence merely for the sake of definiteness, here we outline our lines of argument for the particular case of tetraquark mesons of genuinely exotic quark-flavour content, i.e., bound states $T$ of two antiquarks $\bar{q}_{a}, \bar{q}_{c}$ and two quarks $q_{b}, q_{d}$ the flavour quantum numbers $a, b, c, d$ of which are definitely mutually different:

$$
T=\left[\bar{q}_{a} q_{b} \bar{q}_{c} q_{d}\right], \quad a, b, c, d \in\{u, d, s, c, b\} .
$$

The starting point of the derivation of some QCD sum rule is the choice of a convenient set of interpolating operators. This endeavour is non-negligibly facilitated by the observation that (upon application of adequate Fierz transformations) any conceivable tetraquark interpolating operator can be shown to be equivalent to a sum of products of colour-singlet quark-antiquark bilinear operators. For our study, the spinor structure of any encountered operator is irrelevant and thus notationally suppressed. Then, our basic operators will be the quark-bilinear currents

$$
j_{\bar{a} b}(x) \equiv \bar{q}_{a}(x) q_{b}(x) .
$$

For any fully flavour-exotic tetraquark (1), only two interpolating currents can be constructed:

$$
\theta_{\bar{a} b \bar{c} d}(x) \equiv j_{\bar{a} b}(x) j_{\bar{c} d}(x), \quad \theta_{\bar{a} d \bar{c} b}(x) \equiv j_{\bar{a} d}(x) j_{\bar{c} b}(x) .
$$

Our ultimate goal is the extraction of predictions, emerging from QCD, for the elementary features of the tetraquark (1), that is, its mass $M$, characterizing the location of its pole, its two decay constants $f_{\bar{a} b \bar{c} d}$ and $f_{\bar{a} d \bar{c} b}$, defined in terms of the two tetraquark currents (3) according to

$$
f_{\bar{a} b \bar{c} d} \equiv\left\langle 0\left|\theta_{\bar{a} b \bar{c} d}\right| T\right\rangle, \quad f_{\bar{a} d \bar{c} b} \equiv\left\langle 0\left|\theta_{\bar{a} d \bar{c} b}\right| T\right\rangle,
$$

as well as its transition amplitudes to quark-flavour-matching two-conventional-meson states.

To this end, it proves advantageous, from the technical point of view, to start such analyses from four-point Green functions of ordinary-meson interpolating operators, i.e., from vacuum expectation values of time-ordered $(\mathrm{T})$ products of four quark-bilinear currents of the kind (2), 


$$
\left\langle\mathrm{T}\left(j(y) j\left(y^{\prime}\right) j^{\dagger}(x) j^{\dagger}\left(x^{\prime}\right)\right)\right\rangle .
$$

From these four-point Green functions, all correlation functions we are interested in then arise via configuration-space pair contractions of quark-bilinear currents (2), namely, the two-point correlator of two tetraquark interpolating currents (3) from the contraction of two such $j$ pairs,

$$
\left\langle\mathrm{T}\left(\theta(y) \theta^{\dagger}(x)\right)\right\rangle=\lim _{\substack{x^{\prime} \rightarrow x \\ y^{\prime} \rightarrow y}}\left\langle\mathrm{~T}\left(j(y) j\left(y^{\prime}\right) j^{\dagger}(x) j^{\dagger}\left(x^{\prime}\right)\right)\right\rangle,
$$

the three-point correlator of one tetraquark and two $j$ currents by just a single pair contraction,

$$
\left\langle\mathrm{T}\left(j(y) j\left(y^{\prime}\right) \theta^{\dagger}(x)\right)\right\rangle=\lim _{x^{\prime} \rightarrow x}\left\langle\mathrm{~T}\left(j(y) j\left(y^{\prime}\right) j^{\dagger}(x) j^{\dagger}\left(x^{\prime}\right)\right)\right\rangle .
$$

Dealing with flavour-exotic tetraquarks, we must discriminate between two quark-flavour topologies characterized by whether the quark-flavour distributions in initial and final state are the same or different and, unsurprisingly, analyze separately $[5,6,8]$ two disjoint categories of processes: those that do not undergo quark rearrangement (Sect. 2) and those that do (Sect. 3), called the flavour-preserving (vulgo "direct") and -reshuffling (vulgo "recombination") cases.

We arrive at an identical conclusion $[5,6,8]$ : Within a perturbative expansion in powers of

$$
\alpha_{\mathrm{s}} \equiv \frac{g_{\mathrm{s}}^{2}}{4 \pi}
$$

(with the strong coupling $g_{\mathrm{s}}$, together with all quark masses, constituting the set of parameters of QCD), the tetraquark-phile Feynman diagrams are necessarily of order $O\left(\alpha_{\mathrm{s}}^{2}\right)$ or higher and
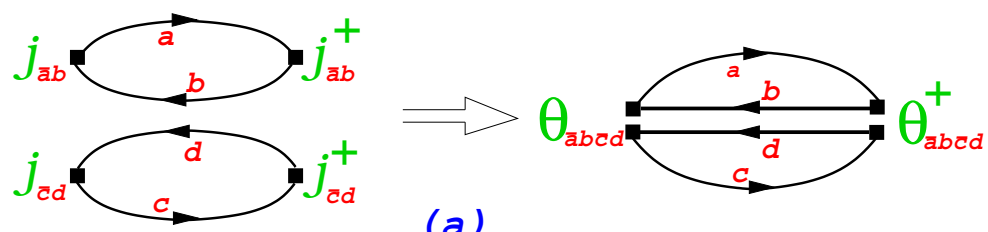

(a)
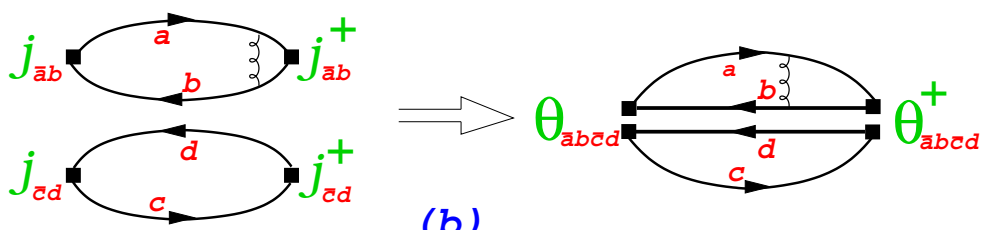

(b)
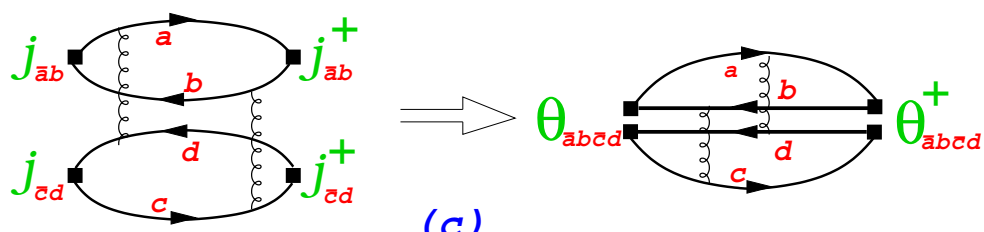

(c)

Figure 1. Typical examples of contributions at the order $O\left(\alpha_{\mathrm{s}}^{0}\right)(\mathrm{a}), O\left(\alpha_{\mathrm{s}}\right)(\mathrm{b})$, and $O\left(\alpha_{\mathrm{s}}^{2}\right)$ (c) of the strong coupling $\alpha_{\mathrm{s}}$ (corresponding to the internal exchanges of no, one or two gluons, respectively, indicated by curly lines) to a flavour-preserving Green function $\left\langle\mathrm{T}\left(j_{\bar{a} b} j_{\bar{c} d} j_{\bar{a} b}^{\dagger} j_{\bar{c} d}^{\dagger}\right)\right\rangle$ of four quark-bilinear currents $j^{(\dagger)}$ (left), and the contraction to the correlator $\left\langle\mathrm{T}\left(\theta_{\bar{a} b \bar{c} d} \theta_{\bar{a} b \bar{c} d}^{\dagger}\right)\right\rangle$ of two tetraquark operators $\theta_{\bar{a} b \bar{c} d}^{(\dagger)}$ (right) $[5,6]$. 
must involve, at least, two gluon exchanges of appropriate non-separable topology, in order to exhibit the requisite four-quark cut in the Mandelstam variable $s$. Consequent implementation of quark-hadron duality renders possible to systematically reject all unambiguously identified ordinary-hadron contamination. Only multiquark-adequate QCD sum rules incorporating and reflecting these insights should be regarded as providing reliable descriptions of multiquarks. ${ }^{1}$

\section{Four-Quark Processes Retaining the Distribution of Quark Flavour}

For four-point Green functions (4) of quark-bilinear operators (2) with identical quark-flavour structure of initial and final states, the proof of our claim is, in fact, rather straightforward, and readily exemplified for the two-point correlators (5) emerging from their contractions (Fig. 1),

$$
\left\langle\mathrm{T}\left(\theta_{\bar{a} b \bar{c} d}(y) \theta_{\bar{a} b \bar{c} d}^{\dagger}(x)\right)\right\rangle, \quad\left\langle\mathrm{T}\left(\theta_{\bar{a} d \bar{c} b}(y) \theta_{\bar{a} d \bar{c} b}^{\dagger}(x)\right)\right\rangle .
$$

Feynman graphs of orders $O\left(\alpha_{\mathrm{s}}^{0}\right)$ and (partly due to vanishing colour traces) $O\left(\alpha_{\mathrm{s}}\right)$ involve two unconnected quark loops: each contributes to the traditional QCD sum rule of its own (Fig. 2).
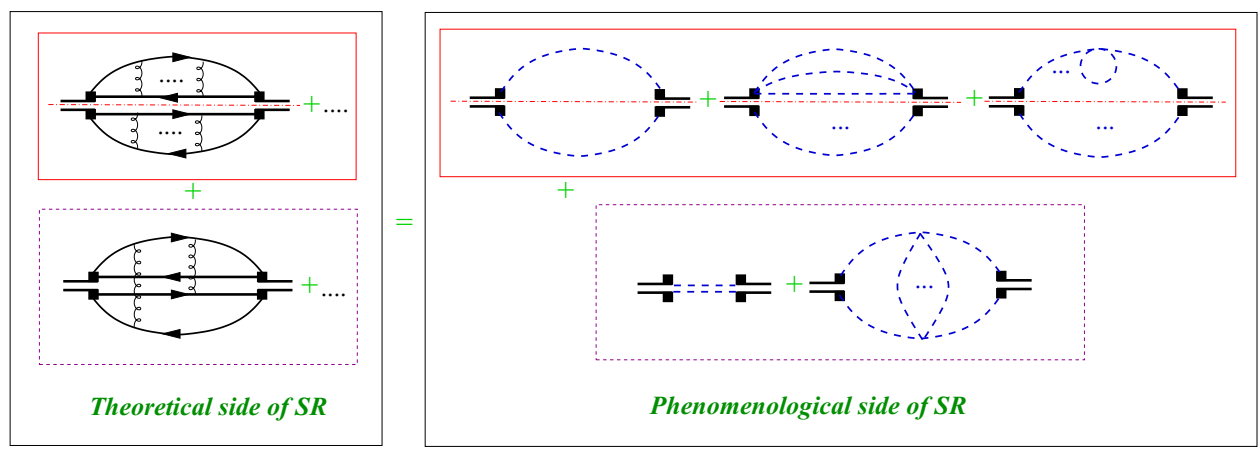

Figure 2. Disentanglement of the QCD sum rule (SR) for any generic flavour-preserving Green function $\left\langle\mathrm{T}\left(\theta_{\bar{a} b \bar{c} d} \theta_{\bar{a} b \bar{c} d}^{\dagger}\right)\right\rangle$ of two, by request flavour-exotic, tetraquark operators $\theta_{\bar{a} b \bar{c} d}^{(\dagger)}$ into a pair of QCD sum rules (indicatively separated by a red horizontal dot-dashed line) for the Green functions of two quark-bilinear currents $j^{(\dagger)}$ interpolating ordinary mesons (generically indicated by blue dashed lines) (top), and a QCD sum rule for the non-separable part of such Green function $\left\langle\mathrm{T}\left(\theta_{\bar{a} b \bar{c} d} \theta_{\bar{a} b \bar{c} d}^{\dagger}\right)\right\rangle$ (bottom). Only the latter can develop a tetraquark pole (blue dashed double line) exhibiting the $\theta_{\bar{a} b \bar{c} d}$ flavour degrees of freedom $[5,6]$.

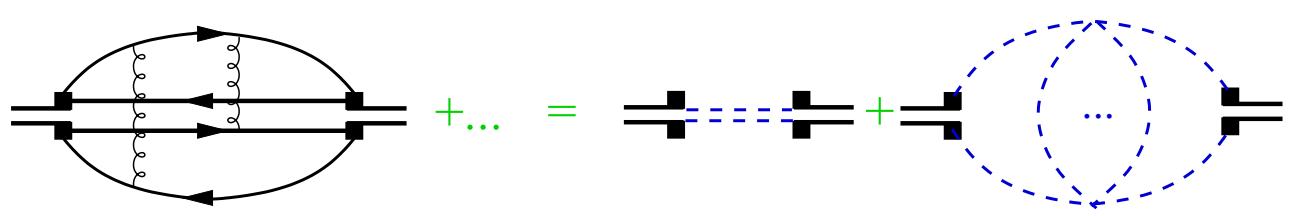

Figure 3. Tetraquark-adequate QCD sum rule for each flavour-retaining Green function $\left\langle\mathrm{T}\left(\theta_{\bar{a} b \bar{c} d} \theta_{\bar{a} b \bar{c} d}^{\dagger}\right)\right\rangle$, relating tetraquark-phile Feynman-diagram contributions [involving at least two gluon exchanges (curly lines), thus of order $O\left(\alpha_{\mathrm{s}}^{2}\right)$ or higher] at the QCD level to contributions of potential tetraquark poles (blue dashed double line) and just non-separable meson diagrams (blue dashed lines) at the hadron level $[5,6]$.

Thus, tetraquark-phile contributions are, at least, of order $O\left(\alpha_{\mathrm{s}}^{2}\right)$. Only these (Fig. 3) should be retained in the construction of QCD sum rules assumed to describe non-conventional hadrons.

\footnotetext{
${ }^{1}$ For pentaquarks too, problems attributable to a too naïve application of QCD sum rules have been noted $[15,16]$.
} 

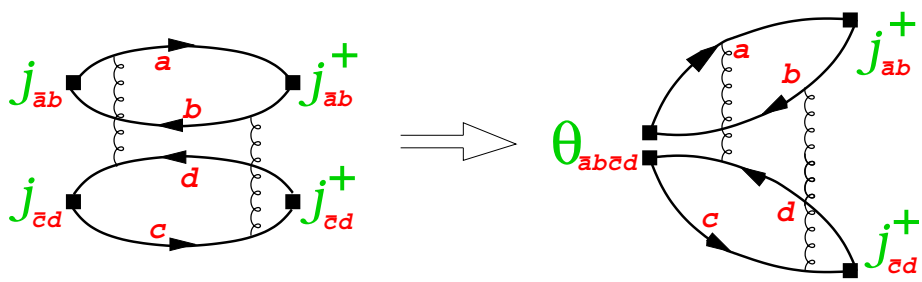

Figure 4. Example of a contribution of lowest tetraquark-phile strong-coupling order $O\left(\alpha_{\mathrm{s}}^{2}\right)$, enabled by exchange of two gluons (curly lines), to the flavour-preserving Green function $\left\langle\mathrm{T}\left(j_{\bar{a} b} j_{\bar{c} d} j_{\bar{a} b}^{\dagger} j_{\bar{c} d}^{\dagger}\right)\right\rangle$ of four quark-bilinear currents $j^{(\dagger)}$ (left), contracted to a correlator $\left\langle\mathrm{T}\left(j_{\bar{a} b} j_{\bar{c} d} \theta_{\bar{a} b \bar{c} d}^{\dagger}\right)\right\rangle$ of a tetraquark current $\theta_{\bar{a} b \bar{c} d}^{\dagger}$ and two quark-bilinear currents $j_{\bar{a} b}$ and $j_{\bar{c} d}$ (right) underlying the tetraquark-two-meson transition $[5,6]$.

The same holds, by its construction (6), for the flavour-retaining (Fig. 4) three-point correlator

$$
\left\langle\mathrm{T}\left(j_{\bar{a} b}(y) j_{\bar{c} d}\left(y^{\prime}\right) \theta_{\bar{a} b \bar{c} d}^{\dagger}(x)\right)\right\rangle .
$$

If stripping off all conventional contributions taken care of by QCD sum rules for ordinary mesons, we are left with flavour-preserving tetraquark-adequate QCD sum rules involving, in their dispersion representation, solely tetraquark-phile spectral densities $\rho_{\mathrm{p}}, \Delta_{\mathrm{p}}$ and the related power corrections, as well as Borel parameters $\tau$, $\tau$-dependent effective thresholds $s_{\text {eff }}$, and, in the case of three-point functions, Fourier transforms $A\left(T \rightarrow j_{\bar{a} b} j_{\bar{c} d}\right)$ of $\left\langle 0\left|\mathrm{~T}\left[j_{\bar{a} b}(y) j_{\bar{c} d}\left(y^{\prime}\right)\right]\right| T\right\rangle$ :

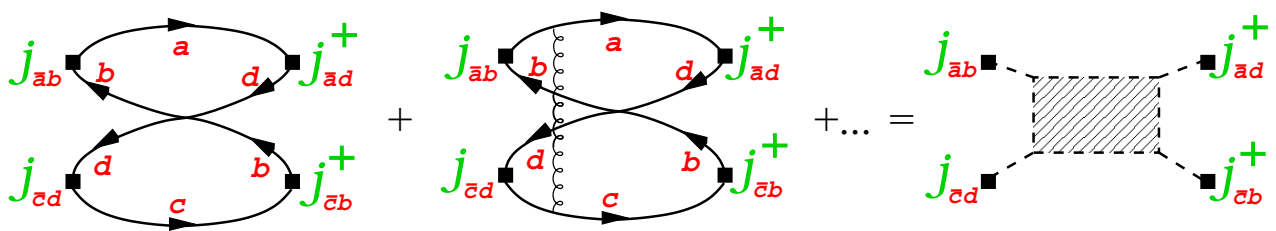

(a)

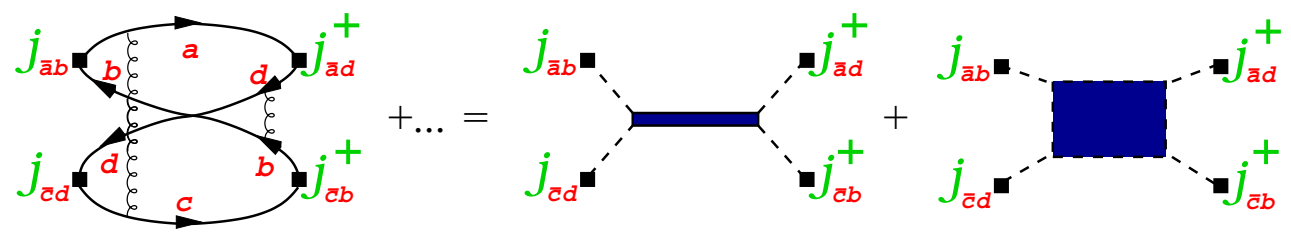

(b)

Figure 5. Disentanglement of the contributions to a flavour-rearranging correlator $\left\langle\mathrm{T}\left(j_{\bar{a} d} j_{\bar{c} b} j_{\bar{a} b}^{\dagger} j_{\bar{c} d}^{\dagger}\right)\right\rangle$ of four flavour-permuting quark-bilinear operators $j^{(\dagger)}$ into (a) QCD-level contributions without four-quark $s$-channel cuts (left), mirrored by hadron-level contributions without two-meson $s$-channel cuts (hatched rectangle, right), and (b) tetraquark-phile QCD-level contributions developing four-quark $s$-channel cuts (left), hence dual to hadron-level contributions (right) encompassing possible tetraquark $s$-channel poles (blue horizontal bar) and contributions comprising a two-meson $s$-channel cut (blue filled rectangle) [8]. 


$$
\begin{array}{r}
\left(f_{\bar{a} b \bar{c} d}\right)^{2} \exp \left(-M^{2} \tau\right)=\int_{\left(m_{a}+m_{b}+m_{c}+m_{d}\right)^{2}}^{s_{\mathrm{eff}}(\tau)} \mathrm{d} s \exp (-s \tau) \rho_{\mathrm{p}}(s)+\text { Borelized power corrections }, \\
f_{\bar{a} b \bar{c} d} A\left(T \rightarrow j_{\bar{a} b} j_{\bar{c} d}\right) \exp \left(-M^{2} \tau\right)=\int_{\left(m_{a}+m_{b}+m_{c}+m_{d}\right)^{2}}^{s_{\mathrm{eff}}(\tau)} \mathrm{d} s \exp (-s \tau) \Delta_{\mathrm{p}}(s)+\text { Borelized power corrections }
\end{array}
$$

\section{Four-Quark Reactions Rearranging the Quark-Flavour Distribution}

For a four-point Green function (4) of quark-bilinear currents (2) with permuted quark-flavour structure of initial and final states, the proof of our claim is a little bit more delicate. Also here, by the Landau equations tetraquark-phile Feynman diagrams start contributing at order $O\left(\alpha_{\mathrm{s}}^{2}\right)$. Quark-hadron duality (Fig. 5) is established by noting that, at hadron level, all members of the set of tetraquark-phile contributions exhibit two-meson intermediate states, whereas all others don't, for both the two-point correlators (5) (Fig. 6) and the three-point correlators (6) (Fig. 7),

$$
\left\langle\mathrm{T}\left(\theta_{\bar{a} d \bar{c} b}(y) \theta_{\bar{a} b \bar{c} d}^{\dagger}(x)\right)\right\rangle \quad \text { and } \quad\left\langle\mathrm{T}\left(j_{\bar{a} d}(y) j_{\bar{c} b}\left(y^{\prime}\right) \theta_{\bar{a} b \bar{c} d}^{\dagger}(x)\right)\right\rangle .
$$
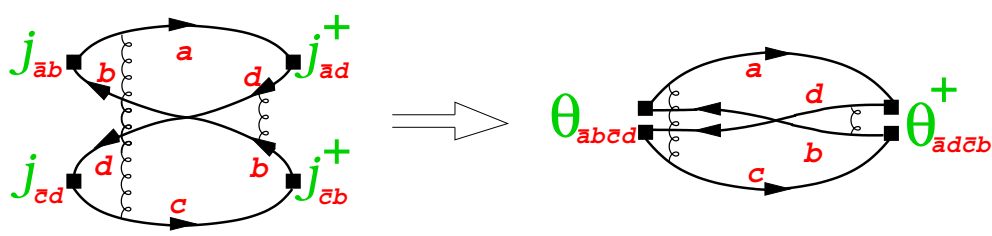

Figure 6. Example of a tetraquark-phile, lowest strong-coupling order- $O\left(\alpha_{\mathrm{s}}^{2}\right)$, two-gluon-enabled (curly lines) contribution to flavour-permuted correlators $\left\langle\mathrm{T}\left(j_{\bar{a} d} j_{\bar{c} b} j_{\bar{a} b}^{\dagger} j_{\bar{c} d}^{\dagger}\right)\right\rangle$ of four quark-bilinear currents $j^{(\dagger)}$ (left), contracted to correlators $\left\langle\mathrm{T}\left(\theta_{\bar{a} d \bar{c} b} \theta_{\bar{a} b \bar{c} d}^{\dagger}\right)\right\rangle$ of two tetraquark operators $\theta_{\bar{a} d \bar{c} b}$ and $\theta_{\bar{a} b \bar{c} d}^{\dagger}$ (right) $[5,6,8]$.
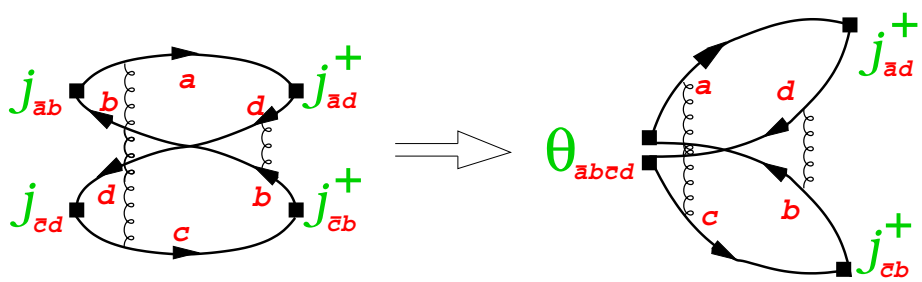

Figure 7. Example of a contribution of lowest tetraquark-phile strong-coupling order $O\left(\alpha_{\mathrm{s}}^{2}\right)$, enabled by exchange of two gluons (curly lines), to a flavour-rearranging Green function $\left\langle\mathrm{T}\left(j_{\bar{a} d} j_{\bar{c} b} j_{\bar{a} b}^{\dagger} j_{\bar{c} d}^{\dagger}\right)\right\rangle$ of four quark-bilinear currents $j^{(\dagger)}$ (left), contracted to a correlator $\left\langle\mathrm{T}\left(j_{\bar{a} d} j_{\bar{c} b} \theta_{\bar{a} b \bar{c} d}^{\dagger}\right)\right\rangle$ of a tetraquark current $\theta_{\bar{a} b \bar{c} d}^{\dagger}$ and two quark-bilinear currents $j_{\bar{a} d}$ and $j_{\bar{c} b}$ (right) underlying the tetraquark-two-meson transition $[6,8]$.

Using our tetraquark-phile spectral densities $\rho_{\mathrm{r}}, \Delta_{\mathrm{r}}$, and Fourier transforms $A\left(T \rightarrow j_{\bar{a} d} j_{\bar{c} b}\right)$ of $\left\langle 0\left|\mathrm{~T}\left[j_{\bar{a} d}(y) j_{\bar{c} b}\left(y^{\prime}\right)\right]\right| T\right\rangle$, we find, as flavour-reshuffling tetraquark-adequate QCD sum rules, 


$$
\begin{array}{r}
f_{\bar{a} b \bar{c} d} f_{\bar{a} d \bar{c} b} \exp \left(-M^{2} \tau\right)=\int_{\left(m_{a}+m_{b}+m_{c}+m_{d}\right)^{2}}^{s_{\mathrm{eff}}(\tau)} \mathrm{d} s \exp (-s \tau) \rho_{\mathrm{r}}(s)+\text { Borelized power corrections } \\
f_{\bar{a} b \bar{c} d} A\left(T \rightarrow j_{\bar{a} d} j_{\bar{c} b}\right) \exp \left(-M^{2} \tau\right)=\int_{\left(m_{a}+m_{b}+m_{c}+m_{d}\right)^{2}}^{s_{\mathrm{eff}}(\tau)} \mathrm{d} s \exp (-s \tau) \Delta_{\mathrm{r}}(s)+\text { Borelized power corrections }
\end{array}
$$

Acknowledgements. D.M. is grateful for support by the Austrian Science Fund (FWF), Project P29028. D.M. and H.S. express gratitude for support under joint CNRS/RFBR Project PRC Russia/19-52-15022.

\section{References}

[1] W. Lucha, D. Melikhov, and H. Sazdjian, Narrow exotic tetraquark mesons in large- $N_{c}$ QCD, Phys. Rev. D 96 (2017) 014022, arXiv:1706.06003 [hep-ph].

[2] W. Lucha, D. Melikhov, and H. Sazdjian, Exotic states and their properties from large$N_{c} Q C D$, PoS (EPS-HEP 2017) 390, arXiv:1709.02132 [hep-ph].

[3] W. Lucha, D. Melikhov, and H. Sazdjian, Tetraquark and two-meson states at large $N_{c}$, Eur. Phys. J. C 77 (2017) 866, arXiv:1710.08316 [hep-ph].

[4] W. Lucha, D. Melikhov, and H. Sazdjian, Are there narrow flavour-exotic tetraquarks in large- $N_{c}$ QCD?, Phys. Rev. D 98 (2018) 094011, arXiv:1810.09986 [hep-ph].

[5] W. Lucha, D. Melikhov, and H. Sazdjian, Tetraquark-adequate formulation of QCD sum rules, Phys. Rev. D 100 (2019) 014010, arXiv:1901.03881 [hep-ph].

[6] W. Lucha, D. Melikhov, and H. Sazdjian, Tetraquark-adequate QCD sum rules, PoS (EPS-HEP2019) 536, arXiv:1908.08803 [hep-ph].

[7] W. Lucha, D. Melikhov, and H. Sazdjian, Cluster reducibility of multiquark operators, Phys. Rev. D 100 (2019) (in print), arXiv:1908.10164 [hep-ph].

[8] W. Lucha, D. Melikhov, and H. Sazdjian, Tetraquark-adequate QCD sum rules for quark-exchange processes, Phys. Rev. D 100 (2019) 074029, arXiv:1909.06324 [hep$\mathrm{ph}]$.

[9] M. A. Shifman, A. I. Vainshtein, and V. I. Zakharov, QCD and resonance physics. Theoretical foundations, Nucl. Phys. B 147 (1979) 385.

[10] K. G. Wilson, Non-Lagrangian models of current algebra, Phys. Rev. 179 (1969) 1499.

[11] W. Lucha, D. Melikhov, and S. Simula, Effective continuum threshold in dispersive sum rules, Phys. Rev. D 79 (2009) 096011, arXiv:0902.4202 [hep-ph].

[12] W. Lucha, D. Melikhov, and S. Simula, Bound-state parameters from dispersive sum rules for vacuum-to-vacuum correlators, J. Phys. G 37 (2010) 035003, arXiv:0905.0963 [hep-ph].

[13] W. Lucha, D. Melikhov, and S. Simula, Extraction of ground-state decay constant from dispersive sum rules: QCD vs potential models, Phys. Lett. B 687 (2010) 48, arXiv: 0912.5017 [hep-ph].

[14] L. D. Landau, On analytic properties of vertex parts in quantum field theory, Nucl. Phys. 13 (1959) 181.

[15] Y. Kondo, O. Morimatsu, and T. Nishikawa, Two-hadron-irreducible QCD sum rule for pentaquark baryon, Phys. Lett. B 611 (2005) 93, arXiv:hep-ph/0404285.

[16] T. Nishikawa, Y. Kondo, O. Morimatsu, and Y. Kanada-En'yo, Pentaquarks in QCD sum rules, Prog. Theor. Phys. Suppl. 168 (2007) 54. 